\title{
Person-Oriented Methods in Partner Violence Research: Distinct Biopsychosocial Profiles Among Battered Women
}

\author{
Paula S. Nurius ${ }^{1}$ and Rebecca J. Macy ${ }^{2}$ \\ ${ }^{1}$ School of Social Work and Harborview Injury Prevention and Research Center affiliate, \\ University of Washington \\ ${ }^{2}$ School of Social Work, University of North Carolina at Chapel Hill
}

\begin{abstract}
Violence researchers have called for the use of person-oriented methods to understand differences that have been found in biopsychosocial consequences among those who experience intimate partner violence (IPV). To address this issue, we apply a person-oriented statistical method, latent profile analysis (LPA), to test for meaningful subgroups of a sample of 448 battered women based on participants' appraisals of their vulnerability relative to their violent partner, depressive symptoms, physical injuries, overall physical health functioning, and their positive and negative social relationships with friends and family. The LPA established five significantly distinct subgroups. Using MANOVA, we examined these subgroups and their respective IPV exposure, both concomitant and separate incidents within the past year. Those with the most intensive violence exposure show the greatest level of challenge and impairment. However, the groups with comparable levels of IPV exposure manifest distinctly different configurations of biopsychosocial profiles, indicating a need for adaptive interventions commensurate with these profiles. We discuss the implications these findings have for developing adaptive interventions for battered women, as well as the potential utility of person-oriented tools for violence researchers.
\end{abstract}

\section{Keywords}

domestic violence; battered; person-oriented; health; coping

\begin{abstract}
In recent decades, the burgeoning research on intimate partner violence (IPV) has amassed a robust collection of evidence that has revolutionized our understanding of the prevalence and seriousness of this endemic societal problem (e.g., Campbell, 2002; Golding, 1999; Logan, Walker, Cole, \& Lukefeld, 2002; Plitcha, 2004; Plitcha \& Falik, 2001; Tjaden \& Thoennes, 2000, 2006; Tolman \& Wang, 2005). The bulk of this research used variableoriented methodologies that yielded an increasingly sophisticated aggregate depiction of IPV, including its epidemiology, factors associated with IPV exposure, and the relationship of IPV exposure to survivors' biopsychosocial well-being. Although more limited, research findings have also shown that community and professional interventions can curb the perpetration of IPV and reduce its negative effects (e.g., Bybee \& Sullivan, 2002; Wasco et al., 2004). These research efforts have revealed the underlying complexities of the IPV phenomenon, including considerable disparateness in the experience of and response to IPV.
\end{abstract}

(C) The Author(s) 2010

Corresponding Author: Paula S. Nurius, 4101 15th Ave NE, School of Social Work, University of Washington, Seattle, WA 98105, nurius@u.washington.edu.

Declaration of Conflicting Interests

The authors declared that they had no conflicts of interests with respect to their authorship or the publication of this article. 
Based on these observations, researchers and clinicians have called increasingly for future IPV research to include person-oriented methods as a way to investigate such heterogeneity and refine the understanding of differences among those who experience IPV (see, for example, Bogat, Levendosky, \& von Eye, 2005; Nurius \& Macy, 2008). Those interested in IPV hold that findings from person-oriented methods will better guide the development of tailored, adaptive community and service responses (Collins, Murphy, \& Bierman, 2004). The study presented here addressed the call for person-oriented methods in IPV research.

In this article, we first review distinctions between variable-oriented and person-oriented methods, which include the utility of person-oriented methods for investigating the dimensions of heterogeneity observed in IPV. Next, we describe our application of latent profile analysis (LPA), which is a person-oriented statistical method, to a sample of battered women. We conclude by discussing the implications of the LPA findings for service development as well as future research efforts.

\section{Distinguishing Person-Oriented and Variable-Oriented Methods}

Variable-oriented methods are based on the proposition that samples are homogenous, or at least sufficiently similar, to allow collected research to be generalized across populations. The social and behavioral sciences have relied on these methods as the workhorses of quantitative analysis to provide a rich foundation of empirical evidence. Variable-oriented methods include many familiar analytic tools, such as analysis of variance and regression that yield parameter estimates based on aggregate information obtained from a study sample and that describe relationships among variables. A key concept in such analyses is the construct of ordinary least squares in which the statistical results are presented as a model that parsimoniously minimizes the sum of the squared deviations of the data. Thus the linear model characterizes the sample overall and describes the relationships among the variables of interest. Variable-oriented tools offer powerful analytic options and provide important information about the statistical relationships among variables of persons in the sample.

In contrast, person-oriented research is based on propositions that distinct subgroups exist in any given sample and that aggregate-level approaches contradict or obscure theoretically and substantively meaningful subgroup characteristics (von Eye \& Bogat, 2006). To investigate distinct patterns of relationships that are shared within a subgroup, personoriented statistical methods determine relationships at the person-level rather than the variable-level. In addition, person-oriented methods are distinct from variable-oriented approaches because person-oriented methods emphasize the potential uniqueness of individuals, including a person's capacity to act on his or her own and the critical need to understand how a person evolves as a whole over time (Bergman \& Trost, 2006). Although person-oriented research is not new, this approach is now being used more often as a result of improved methods, availability of relevant statistical software for analysis, and recognition that these methods address the need for information related to complexity and heterogeneity that is not readily accomplished using variable-oriented methods (Curran \& Willoughby, 2003; Muthen, 2002; von Eye, Bogat, \& Rhodes, 2007).

Although interest in person-oriented research has been found most often in developmental psychology, increasing interest in this research approach has emerged in the interpersonal violence field, and particularly in the area of intimate partner violence (Bogat et al., 2005; Hughes \& Huth-Brocks, 2007; Kohl \& Macy, 2008; Macy, 2008; Nurius \& Macy, 2008). These discussions of methodology are linked to growing documentation of diversity among survivors of intimate partner violence. For example, Briere and Jordan (2004) concluded that "the effects of interpersonal violence vary substantially from person to person and cannot be defined by preformulated assault syndromes or lists of expected symptoms" (p. 
1267). Such discussions not only point to the critical need for tailored, adaptive interventions (Collins et al., 2004) that address the varied needs of violence survivors but also underscore the need for additional person-oriented research to provide a framework of empirical evidence to support and inform the development of such interventions.

In sum, the results of variable-oriented methods reflect relationships between the dependent and independent variable(s), aiming to characterize the relationships between the variables of interest for the sample, overall. Quantitative person-oriented methods, which are our focus here, are designed for different kinds of questions; complementary to what variableoriented methods provide. Whereas both approaches use "variables" in the form of quantitatively derived data, person-centered strategies test for intraand interindividual differences among the variables of interest. The results of person-oriented statistical methods are patterns of characteristics among multiple variables that one subgroup hold in common and that distinguish them from other subgroups.

\section{Latent Variable Approach With Battered Women}

An important challenge for those who want to use person-oriented research methods in IPV investigations is deciding which approach will provide the meaningful results with the greatest relevance for domestic violence service providers and their service systems. Although there are numerous approaches to person-oriented research (Bergman, 2000; Bergman \& Trost, 2006; Bergman, von Eye, \& Magnusson, 2006; Curran \& Willoughby, 2003), person-oriented, latent variable statistical tools can be fruitfully used to test for patterns among subgroups based on multiple variables simultaneously (Bollen, 2002; Hagenaars \& Halman, 1989; Muthen, 2002).

This multivariate capacity allows researchers to examine violence-related factors in combination. Although the IPV literature has long recognized diversity among victims and the need for differential responding (Davies, Lyon, Monti-Catania, 1998; Lindhorst, Nurius, \& Macy, 2005), there has been limited empirical guidance at the multivariate level as to how needs differentially cluster. Similar to the way that factor analysis helps search for factor structure within a set of variables, person-oriented latent variable statistics identify substantively important subgroups within a study sample based on similar patterns of characteristics (Muthen, 2002). Thus the statistical model produced by a person-oriented latent variable analysis provides results based on (a) whether distinct groups are present, (b) the number of distinct groups, (c) the prevalence and size of the groups, (d) which participants are classified into which groups, (e) the patterns of variables that distinguish each group, and (f) model fit (Macy, 2008; Muthen, 2002; Nurius \& Macy, 2008).

As with any statistical analysis, the critical selection of variables included in the analysis should be made based on substantive or theoretical reasoning (Muthen, 2002). In the present study, we examined factors expected to affect battered women's capacity to cope with abuse and to seek safety in the immediate aftermath of an IPV incident. The goal of personoriented methods is not to investigate all factors with potential relevance to the target phenomenon but rather to select indicators across domains of factors that may conjointly influence the outcome of interest. With this goal in mind, we investigated biopsychosocial factors that have been shown as empirically related to women's capacity for self-protective coping in the face of IPV. Moreover, these biopsychosocial factors can be bolstered through domestic violence services. We investigated six factors associated with survivors of IPV: (a) survivors' appraisals of their vulnerability relative to their violent partner (i.e., their perceptions of susceptibility to physical and psychological danger from their partners), (b) depressive symptoms, (c) physical injuries, (d) overall physical health functioning, (e) positive social relationships, and (f) negative social relationships with friends and family. 
We chose to investigate survivors' appraisals of their vulnerability from their partners because stress and coping theory has repeatedly been applied to understanding the dynamics of women's IPV exposure. Consistent with stress and coping theory, IPV is a significant stressor and threat to women's well-being. However, each individual appraises and copes with the threat of IPV in a unique biopsychosocial context (Lindhorst et al., 2005; Sutherland, Bybee, \& Sullivan, 2002). For example, the appraisal dimension of stress and coping theory emphasizes that individuals may react differently to the same stressor based on their personal interpretations of the stressor (Lazarus \& Folkman, 1984). Also consistent with stress and coping theory, research has shown that women's appraisals and attributions of IPV are associated with their symptomatology, coping efforts, and willingness to seek help (Henning \& Klesges, 2002; Hutchison \& Hirschel, 1998; Macy, Nurius, Kernic, \& Holt, 2005; Nurius et al., 2003; Pape \& Arias, 2000). However, full understanding of survivors' appraisals, coping styles, and help-seeking efforts cannot be determined by assessing IPV acts alone. Researchers have also pointed to women's sense of entrapment, susceptibility to harm, and feelings of disempowerment as key factors in understanding survivors' appraisals and coping efforts (Nurius et al., 2003; P. H. Smith, Thornton, DeVellis, Earp, \& Coker, 2002).

Reports from recent research efforts have included dense documentation of IPV as a negative stressor for women's physical and mental health (Campbell, 2002; Golding, 1999; Logan et al., 2002; Plitcha, 2004). Depression, which is one of the most commonly reported indicators of poor mental health, is elevated with abuse and is negatively related to both adaptive coping and use of coping resources such as social support (Carlson, McNutt, Choi, \& Rose, 2002; Hathaway et al., 2000; Hazen, Connelly, Kelleher, Landsverk, \& Barth, 2004; Kramer, Lorenzon, \& Mueller, 2004; Porcerelli et al., 2003; Zlotnick, Johnson, \& Kohn, 2006). IPV-related injuries and poor physical health compromise survivors' daily functioning, quality of life, and ability to engage in career, work, family, and relationship roles (Carbone-Lopez, Kruttschnitt, \& Macmillan, 2006; Coker, Smith, Bethea, King, \& McKeown, 2000; Coker, Smith, \& Fadden, 2005; Tolman \& Wang, 2005). However, both physical and mental health outcomes for survivors improve when their exposure to violence ceases (Golding, 1999; McNutt, Carlson, Persaud, \& Postmus, 2002; Tolman \& Rosen, 2001).

Survivors' social support is typically understood as buffering the impact of partner violence on physical and mental health and improving coping, and, therefore, such support is usually studied as a protective factor (Carlson, McNutt, et al., 2002; Coker, Watkins, Smith, \& Brandt, 2003). However, not all social relationships offer protective potential as is evidenced by the negative social relations experienced by battered women (Nurius et al., 2003). Thus we included both negative and positive social relationships in our analysis.

\section{Method}

\section{Sample and Recruitment}

The sample used in this study included 448 adult women (18 years or older) from Seattle, Washington, who were victims of abuse by a current or former intimate partner that resulted in a police-reported incident or filing of a protective order (see Wolf, Holt, Kernic, \& Rivara, 2000 for detailed information). The sample was selected based on a stratified random sample of reports and protective orders filed within a 13-month period and based on the women's actions to involve the legal system, which constituted the index episode of abuse for each participant. Of the 742 women contacted, 108 (14.6\%) could not be located, $124(16.7 \%)$ declined to participate, $62(8.4 \%)$ agreed but did not complete the interview, and 448 (60.4\% of the total; $70.7 \%$ of those located) completed the interview. Participants and nonparticipants were similar on age, marital status, proportion having a child with the 
abuser, type of offense reported to the police, and proportion injured at the index incident. Participants were more likely than nonparticipants to have obtained protection orders and less likely to be living with the abuser at the time of the index event (Macy et al., 2005).

The University of Washington's Human Subjects Review Committee approved study protocols. Approximately 1 month after the index incident, eligible study participants were contacted by telephone and asked to participate in the study. Those who agreed to participate were asked to complete a baseline questionnaire and three follow-up questionnaires. Participants were given the option of completing the interviews via telephone (i.e., interviewer administered) or receiving a copy of the survey via U.S. mail (i.e., selfadministered survey). The majority of participants (81\%) chose the telephone interview, and no significant differences have been detected as a function of survey method. The index event enabled this investigation to recruit a diverse sample of battered women at relatively comparable points in their current violence exposure (e.g., sufficient to trigger police reporting). The current analysis focuses on baseline and year prior experiences.

At the time of the baseline interview, participants' ages ranged from 18 to 70 years $(M=$ $32.01, S D=9.46)$. Slightly over half of the sample described themselves as White (54.3\%); $21.5 \%$ self-identified as African American; $7.2 \%$ as Asian/Pacific Islander; $4.3 \%$ as Native American/Alaska Native; $25 \%$ as Hispanic/Latino; and 5.9\% as biracial or multiracial. Participants reported a wide range of educational attainment: eighth grade or less (2.2\%), some high school (9.2\%), high school graduate/GED (24.6\%), post-high school training but not college (7.8\%), some college (37.5\%), college graduate (15.4\%), and advanced/graduate degree $(3.3 \%)$. Similarly at the baseline interview, participants reported a range of occupational statuses: employed full-time (48.9\%), employed part-time (12.3\%), attending school (3.8\%), both attending school and employed (9\%), and not employed outside the home (25.3\%). Less than $1 \%$ of the sample reported being either self-employed or disabled. Although the largest percentage of participants reported household income of less than US $\$ 15,000$ per year (43.9\%), all income levels were reported: US $\$ 15,000$ to US $\$ 19,999$ (11.8\%); US $\$ 20,000$ to US $\$ 24,999$ (8.3\%); US $\$ 25,000$ to US $\$ 34,999$ (14.5\%); US $\$ 35,000$ to US $\$ 49,999$ (12\%); US\$50,000 to US\$69,000 (4.9\%); and US\$70,000 and greater (4.7\%). Less than $5 \%$ of the sample self-identified as non-U.S. citizens.

\section{Measures}

Data were collected using survey questionnaires that followed a structured format developed through combining several well-evaluated measures described below.

Vulnerability appraisals-Developed as a supplement to behavioral measures, the Women's Experiences with Battering scale (WEB) captures variability in how women appraise and experience violence. The WEB measures psychological vulnerability by assessing women's perceptions of susceptibility to physical and psychological danger, loss of power, and loss of control in a relationship with a male partner (P. H. Smith et al., 1999; P. H. Smith, Earp, \& DeVellis, 1995). The WEB has demonstrated high internal consistency, good construct validity, and significant correlations with known-group status. Responses to the 10 WEB items were given using a 6-point Likert-type scale ranging from 1 (strongly disagree), to 6 (strongly agree); responses were totaled to create a summary score $(M=39.35, S D=16.44, a=.94)$.

Depression-To assess participants' depressive symptoms, the survey instrument included the Center for Epidemiologic Studies of Depression Scale (CES-D). Participants were asked to base their responses on the week immediately preceding the interview. The 20 items were rated on a 4-point Likert-type scale ranging from 0 (rarely or none of the time), to 3 (most or 
all of the time); responses were totaled to create a continuous measure $(M=32.44, S D=$ $14.74, a=.95)$. A CES-D score of 16 or less indicates no depression, a score of 17 to 26 indicates mild depression, and a score of 27 or greater indicates severe depression.

Injuries at incident-Participants were asked to indicate if the index incident of partner abuse caused any of the following six types of injuries: pain, bruises and/or soreness or swelling, cuts and/or bleeding, broken bone(s), internal injury or injuries, or loss of consciousness. The positive responses to each item were totaled to provide an injury index $(M=1.19$, range $=0-6)$.

Physical functioning-The standardized summary score for the physical health component (PCS-12) of the Short Form Health Survey (SF-12) was used to measure each participant's overall level of physical functioning (e.g., level of activity, ability to get things accomplished; $M=46.66, S D=10.68$ ). The reliability and validity of SF-12 has been extensively investigated and discussed elsewhere (Ware et al., 1995). Indicator variables of 0 and 1 are created from the response choice categories, weighted using regression coefficients from the general U.S. population, and aggregated to allow comparison of results across studies. Among U.S. women, the mean PCS-12 score is 49.11 with a standard deviation of 9.92. Lower scores on the PCS-12 are indicative of poorer physical health status.

Social relations-positive and negative-Social relations were measured using items from the Social Adjustment Scale (SAS-M) that pertained to engagement with friends and family members (Cooper, Osborn, Gath, \& Feggetter, 1982; Weissman, Prusoff, Thompson, Harding, \& Myers, 1978). Cooper and colleagues (1982) adapted the original SAS (Weissman et al., 1978) to use a 5-point Likert-type scale ranging from 1 (not at all), to 5 (all the time) to rate all items. Seven items were clustered into a set for positive social support (e.g., "Been able to talk about your feelings openly with your friends," "Depended on your relatives for help, advice, or friendship") and nine items comprised the negative social relations set (e.g., "Gotten angry with or argued with your friends," "Been feeling that your relatives had let you down"). Each set was averaged to yield either a positive social relation scale $(M=2.75, S D=.98, \alpha=.80)$ or a negative social relation scale $(M=2.01, S D$ $=.67, \mathrm{a}=.69)$.

Intimate partner violence exposure-Assessments of the severity of physical, sexual, and psychological abuse the participant experienced in the year prior to the index incident as well as at the incident that triggered legal involvement were measured using subscales from the revised Conflict Tactics Scale (CTS2; Straus, Hamby, Boney-McCoy, \& Sugarman, 1996). Consistent with the CTS2 guidelines, respondents indicated the frequency with which they experienced various types of partner violence. We constructed subscales by summing each participant's responses for psychological abuse ( 8 items, range $=0-200 ; M=60.08 ; \mathrm{a}$ $=.83$ ), physical abuse during the prior year (12 items, range $=0-234 ; M=24.25 ; \alpha=.90)$, and sexual abuse ( 2 items, range $=0-50 ; M=5.31 ; \alpha=.59$ ). Participants provided responses to a nearly identical set of items asked for the index event; however, instead of indicating a frequency of abuse, participants indicated whether that type of abuse occurred at the incident ( 1 = occurred; $0=$ did not occur); responses were totaled to create the psychological abuse scale (range $=0-8 ; M=3.65 ; \alpha=.70$ ), the physical abuse scale (range $=0-12, M=2.82 ; \mathrm{a}$ $=.85$ ), the sexual abuse scale (range $=0-2, M=.15 ; a=.41$ ).

We note that (a) vulnerability appraisals, (b) depression, (c) injuries at incident, (d) physical health functioning, and (e) social relations - both positive and negative-were primarily selected for inclusion in the LPA analysis based for the substantive and theoretical reasons 
discussed early. In addition and prior to the LPA analysis, we examined the bivariate correlations among these variables as well as between each of these variables and the IPV exposure variables. As anticipated, these analyses showed statistically significant relationships among the variables. Nonetheless, no statistical relationship was so strong (i.e., .7 and higher) to indicate that we had measured similar constructs with these various measures.

\section{Results Analysis}

Latent profile analysis (LPA), which is a cluster analysis technique that uses maximum likelihood procedures, was used to determine whether the sample contained distinct subgroups of participants with unique patterns of the bio-psychosocial variables of interest (Everitt, Landau, \& Morven, 2001; Gibson, 1959; Muthen, 2002). If the analysis identifies groups with unique variable patterns, then each group can be assumed to have come from a population with a separate probability distribution (Everitt et al., 2001). Cleland, Rothschild, and Haslam (2002) found this kind of statistical analysis yielded an accurate classification in samples of 300 or more participants.

We used the Mplus 3.0 software to perform the LPA. We ran multiple solutions and identified the optimal number of groups by using the substantive meaningfulness of the models and three-model fit statistics: the Bayesian Information Criterion (BIC), the LoMendell-Rubin (L-M-R) test statistic, and probability statistics (Everitt et al., 2001; Muthen, 2002). Of all the solutions, the five-profile group model had the lowest BIC value $(13,888.67)$, which indicated the best model fit. In conjunction with the BIC values, we also examined the L-M-R statistic, which is an indicator of how many groups should be extracted from the dataset. This statistic compares the parsimony of the current model against the model with one less group; the five-profile model solution was significant $(79.13, p<.01)$. Last, we examined the probability statistics, which show how well individual participants are classified into one group or another based on a given model as well as the utility of the model in differentiating groups. Probability statistics can be thought of as similar to factor loadings (Muthen, 2002). The probability statistics for groups one through five in this model were $.94, .84, .94, .91$, and .87 , respectively. Given the low BIC value, the fact that the L-M$\mathrm{R}$ statistic was significant, and the strong probability statistics for the five-group profile solution, we examined this solution for its substantive usefulness and found it to be robust.

\section{Profile Structures}

Table 1 presents our LPA group findings and reports the means and standard deviations for the biopsychosocial variable indicators for each group. Figure 1 shows these means in standardized form. We labeled each profile to identify the key substantive features of the group. Profile $1(n=68)$, which we labeled as Multiple Resources, was distinct from the overall sample and other profile groups in that this group of women reported relatively good physical health, less difficulty with depression, lower vulnerability appraisals, and high positive social support. Women in Profile 2, Struggling With Depression $(n=69)$, were distinguished by their relatively high levels of depression with lower vulnerability appraisals. The third profile group, Vulnerable-Supported $(n=58)$, was characterized by vulnerability appraisals that were very close to the overall sample mean but with relatively high positive social relationships and relatively less difficulty with physical health and depression. The fourth profile, Vulnerable-Depressed $(n=160)$, which was the largest group in the sample, showed a profile with the second-highest level of vulnerability appraisals as well as higher than average depression. The fifth profile, Severe Functioning Impairment (n =93), was the second-largest subgroup in the sample. These women exhibited a consistent 
pattern of high levels of injury, depression, vulnerability appraisals, and negative social relations in addition to lower levels of both physical functioning and positive social support.

Tests of group differences were undertaken on sociodemographic and relationship characteristics to investigate relationships between victims' biopsychosocial profiles and their personal and contextual characteristics. No significant group differences were found on race, age, income, education, employment, citizenship, marital status, or relationship duration with abuser (Nurius \& Macy, 2006).

\section{Partner Violence Exposure Differences}

Subsequent to LPA, we examined the groups in relation to the type and severity of IPV experienced at the index incident and in the year before the index incident. Table 2 shows the means and standard deviations for each profile group on psychological, physical, and sexual violence in these two periods. We used MANOVA to test for statistically significant differences among the profile groups on IPV exposure. MANOVA is a conservative statistical approach that tests multiple outcomes simultaneously and, thereby, avoids the risk of an inflated overall Type I error rate from using multiple univariate tests (Stevens, 1996). The violence exposure variables were grouped together in time-ordered sequence so that the past year partner violence variables were tested together and the index incident partner violence variables were tested together (see Table 2). Wilks's Lambda was used to test the multivariate null hypothesis that there would be no difference among the groups on partner violence exposure in the past year and at incident. Analyses of variance were applied to findings of significant MANOVA tests to determine which profile groups did and did not significantly differ on each form of partner violence exposure (Table 2).

All MANOVA and ANOVA results indicated statistically significant overall group mean differences at index incident and over the prior year on all forms of violence exposure. Tukey post hoc tests were applied to specific group-to-group comparisons. Relative to violence exposure at the index incident, the first three profile groups (i.e., Multiple Resources, Struggling With Depression, Vulnerable-Supported; see Table 2 and Figure 2) did not significantly differ on any form of abuse. The same was largely true for violence exposure over the past year (Figure 3), with the exception that women in the VulnerableSupported group (Profile 3) had experienced more psychological abuse relative to women in the Multiple Resources group (Profile 1). However, even though these women had highly comparable violence exposure, the three groups manifested very different profiles (Figure 1). The Severe Functioning Impairment group (Profile 5) stood in sharp contrast with the significantly highest levels of exposure to all forms of violence, both at index incident and the prior year. Violence exposure also differed between two other groups: the VulnerableDepressed (Profile 4) group violence exposure at index incident was significantly greater than that of the Multiple Resources (Profile 1) group, which had the least exposure to violence. However, Profiles 2, 3, and 4 were equivalent on exposure to violence (Figure 2). This pattern of violence exposure was consistent and characterized the violence exposure of these groups during the prior year, with the addition of Vulnerable-Depressed (Profile 4) women who experienced greater psychological abuse relative to the Struggling With Depression (Profile 2) group (Figure 3).

\section{Discussion}

Focusing on IPV-abused women, this investigation illustrates the usefulness of personoriented analytic tools as an innovative way to think of, test for, and examine subgroups of violence survivors. Extending violence research that stresses the importance of considering multiple biopsychosocial factors in combination, our analyses established five substantively meaningful subgroups of women. These subgroups had distinct, yet coherent, structures of 
biopsychosocial factors that have been empirically related to women's capacity for selfprotective coping in the face of IPV and have been shown to be bolstered through domestic violence services. It is noteworthy that the findings also show that some subgroups experienced similar levels of IPV, whereas other groups differed significantly on level of violence exposure. The results reveal distinct patterns within the biopsychosocial heterogeneity among IPV abused women to which we now turn.

\section{Profile Structure Distinctions}

Multiple Resources-The group defined under Profile 1, Multiple Resources, reflected the highest level of social support and functioning in the sample. This group's levels of depression, vulnerability appraisals, and negative social relations were markedly lower than the other groups, on average. These women's physical functioning was slightly higher (53.12 vs. 49.11) than the national average and the only group whose average depression scores indicated a nonclinical level (Ware et al., 1995). Women in this group had a very favorable ratio of positive to negative relations, which indicated that social support was a strong asset. In short, these women reported greater internal and external resources at their disposal, which distinguished them in varying ways from the Struggling With Depression (Profile 2) and Vulnerable-Supported (Profile 3) women who reported similar exposure to violence. Although the mean level experiences of partner abuse among women in the Multiple Resources group was lower than that of the women in Struggling With Depression and Vulnerable-Supported groups, these differences were nonsignificant at the index incident and were largely nonsignificant in what these three groups experienced over the prior year. Thus, as illustrated in Figure 3, exposure to violence alone cannot account for the differing arrays of biopsychosocial assets and impairments of abused women in the first three groups (i.e., Multiple Resources, Struggling With Depression, and VulnerableSupported).

Although the women with the Multiple Resources profile appeared to be at less risk of trauma development, their index experience with partner violence was sufficiently serious to warrant legal involvement. The favorable levels of positive versus negative relations found at the subgroup level suggested that the presence of social support and lack of socially undermining relationships provided protective functions for the women in the Multiple Resources group. Thus safety-planning services that seek to reinforce supportive relationships, to educate women in understanding partner violence, and to emphasize the importance of sustained support appeared to be appropriate for this subgroup of women and may have helped reduce the risk of escalated experiences of partner violence and trauma development (Lindhorst et al., 2005; Seng, 2002).

Vulnerable-Supported-The biopsychosocial profile of women in the VulnerableSupported group was largely similar to that of women in the Multiple Resources subgroup in regard to physical functioning, limited injuries at incident, and very favorable positive-tonegative social relationship resources. Although the depression level of VulnerableSupported women indicated a mild depression, it was their higher levels of appraisals of vulnerability from their abuser that most distinguished the Vulnerable-Supported from the Multiple Resources group profiles. Moreover, the higher level of perceived vulnerability among Vulnerable-Supported women significantly paralleled their greater exposure to psychological abuse over the prior year, relative to the Multiple Resource subgroup. However, the Vulnerable-Supported group's profile of biopsychosocial factors prompted consideration of differing functions of vulnerability appraisals. Combined with injuries, depression, and social relations that are more negative than positive, high psychological vulnerability is likely to reinforce impairment and blunt women's ability to gain a sense of self-worth, personal agency, or resilience. However, moderate levels of perceived 
vulnerability that are experienced in the absence of depression and coupled with positive supports and stronger physical functioning, may provide a self-protective catalyst that leads women to seek safety. In other words, at some levels and in some combinations, perceived vulnerability may be a risk factor that serves to augment trauma development and revictimization risk. At other levels and in other combinations, perceived vulnerability may serve a protective function that facilitates protective actions such as help-seeking (Macy et al., 2005), which in turn buffers against trauma development.

Thus the Vulnerable-Supported women had several important protective factors to work with and which could be reinforced through receipt of violence services. Similar to the Multiple Resources group and given their experiences with partner violence, women fitting the Vulnerable-Supported biopsychosocial profile are likely to benefit from safety planning and partner violence education services. For example, McFarlane, Parker, Soeken, Silva, and Reel (1998) found that victimized women who worked with professionals to create a safety plan were more likely to follow through with safety-related activities. What needs additional, careful assessment is the function of the Vulnerable-Supported group's perception of vulnerability. The Vulnerable-Supported women experienced significantly greater psychological abuse over the prior year and their higher vulnerability appraisals indicated greater traumatic impact of the violence that shaped their self-perceptions, their beliefs about their ability to control violence their lives, and potentially, their protective behaviors (P. H. Smith et al., 2002). In addition to safety planning and reinforcement of the social support network, women fitting the Vulnerable-Supported profile need assistance with the potentially trauma-inducing effects of perceived vulnerability and powerlessness relative to their aggressor. Without such assistance, their feelings of terror and powerlessness may prevent these women from seeking safety. Whether this level of vulnerability appraisals serves more of a galvanizing or undermining function on women's safety-related activities is yet to be determined and needs explicit attention.

Struggling With Depression-The Struggling With Depression group measured substantially higher on depression, with their average scores in the severe range of the depression scale. However, this profile group reported lower vulnerability than the Vulnerable-Supported group. Furthermore, the Struggling With Depression group reported more numerous injuries at index incident, lower physical functioning, and less favorable positive-to-negative social relations. Notably, this group did not differ significantly from the Multiple Resources group or the Vulnerable-Supported group on any dimension of partner violence exposure. However, women in the Struggling With Depression group reported elevated depression, lower physical functioning, and less favorable social support; all of which may well have interacted to create greater vulnerability to physical and mental health problems, as well as greater risk for future violent victimization. Although their current safety level did not appear different as a function of the behavioral indicators of violence to which they had been exposed, their psychosocial risk and protective factors profile warranted greater mental health and social support intervention relative to the Multiple Resources and Vulnerable-Supported groups of women. The qualitative nature of the social relationships among the Struggling With Depression group of women may also warrant further investigation to assess how these relationships affect the survivors' well-being.

The needs of the Struggling With Depression profile group suggest the importance of making combined mental health and violence-reduction services available for battered women. The women with this biopsychosocial profile have multiple needs that can be addressed through services, including physical injury and problematic negative social relationships. However, their most pressing need is evident in their struggle with depressive symptoms, which, as discussed earlier, are prevalent among battered women and are likely to impede women's ability to take self-protective actions. Equally important, battered 
women's difficulties with depression are likely to diminish when partner violence stops, which suggests a reciprocal and dynamic relationship between violence and depression. Thus the combination of safety and mental health services may be critical for women struggling with both partner violence and depression.

These risk and protective profiles of the women in the Vulnerable-Depressed group bore similarities to those of the Struggling With Depression group; that is, both groups of women reported severe depression, moderate physical functioning, and moderate social support. However, one factor that sharply distinguished the Vulnerable-Depressed group from others was the former's high level of appraised vulnerability to their abuser, which was more than double that of the Struggling With Depression group. Notably, these two groups did not differ significantly on any aspect of violence exposure at the index incident, nor did they differ on the frequency of physical abuse experienced in the prior year. However, the women in the Vulnerable-Depressed group reported significantly greater levels of psychological abuse in the prior year than did the Struggling With Depression group of women. In addition, the level of sexual abuse experienced by the Vulnerable-Depressed group in the past year approached significance. This finding is congruent with those for the Vulnerable-Supported profile women, wherein elevated vulnerability appraisals were found in the context of greater psychological abuse.

The multidimensional biopsychosocial need of the Vulnerable-Depressed group underscores the importance of comprehensive services that combine safety planning with mental and physical health services (Riger, Raja, \& Camacho, 2002). Similar to the service recommendations for the women classified as Struggling With Depression, the women fitting the Vulnerable-Depressed biopsychosocial profile are more likely to need help with their physical health, mental health, and safety. Services that focus on one problem are likely to be less effective overall than services that attend to women's multiple needs in combination. Akin to women in the Severe Functioning Impairment group, the women in the Vulnerable-Depressed group had exceptionally high levels of appraised vulnerability that involved deeply ingrained, enduring experiences of vulnerability, loss of power and control, and entrapment that carried serious risks for future victimization risk and deepening trauma development (S. P. Smith, Edwards, \& DeVillis, 1998).

Severe Functioning Impairment-This group was by far the most gravely impaired. They sustained more severe injuries at the index incident, reported a substantially lower level of physical functioning than the national norm (35.83 vs. 49.11), suffered with severe levels of depression, as well as high levels of vulnerability appraisals, and were the only group whose negative social relations outstripped their positive social relations. The Severe Functioning Impairment group described relationships with friends and family members that were predominantly characterized by strife and lack of dependable support. This lack of support is especially worrisome given recent work that has posited that, rather than a "flight or fight" response, women tend to rely on positive, supportive relationships in times of stress. This pattern of stress regulation has been labeled "tend-and-befriend" and researchers in this area have argued that women who rely on positive relationships and seek out new supports during times of stress tend to fare better than those who do not (Taylor et al., 2000).

Averages on every dimension of violence exposure, both at the index incident and over the prior year, were significantly higher for the Severe Functioning Impairment subgroup than all other groups. Women fitting the profile of severe impairment have urgent physical health, mental health, and social support needs. Moreover, these women appear to be at greatest risk for subsequent victimization. We cannot ascertain from these data whether the severity of their sustained partner victimization caused an erosion of their biopsychosocial functioning, whether they had a marked risk profile that predated their victimization that 
may have increased their vulnerability, or whether both of these dynamics came into play. However, what was apparent from the data is that these women were in precarious circumstances of having insufficient personal and social resources important to seeking safety or otherwise defending themselves against an abuser.

The severity of violence a woman has experienced has been found to shape her safetyplanning and help-seeking activities, with higher severity corresponding to greater action (Goodkind, Sullivan, \& Bybee, 2004; Macy et al., 2005). This finding suggests that the Severe Functioning Impairment group women would be more likely to seek formal help for partner violence. However, the variable-oriented findings, on which this assumption of helpseeking is based, may or may not apply equally to all subgroup. In particular, we need to ascertain whether this general linear trend applies to those with the highest levels of biopsychosocial impairment or whether active planning and outreach efforts may also be impaired. It is also unclear whether, or to what extent, domestic violence services are equipped to assist women's complex biopsychosocial needs and problems. The finding that those higher functioning clients are also those who report greater satisfaction with counseling and case management in shelter settings (McNamara, Ertl, Marsh, \& Walker, 1997) emphasizes the need to determine how best to deliver services that are tailored to severity of abuse as well as a woman's composite functioning status, need for trauma recovery, and the social context to which she will return. Like the Vulnerable-Depressed group, the women in the Severe Functioning Impairment group also need multidimensional services that are matched to their level of isolation, vulnerability, and impairment.

\section{Survivor Heterogeneity: Implications for Services}

Consistent with Briere and Jordan's (2004) review of violence sequelae variability, the results of the current study elucidate ways in which IPV survivors vary in configurations of their biopsychosocial factors and in interrelations of those factors with violence exposure. It was not surprising to find that the most severely distressed and impaired survivors were those who had experienced exceptionally high levels and multiple forms of intimate partner violent victimization (Campbell, 2002; Kemp, Rawlings, \& Green, 1991). The general linear trend of violence exposure levels during the year prior to data collection (Figure 2) points to the consequential role of proximal violence levels on violence survivors' outcomes. For example, those women with the lowest and highest levels of violence exposure show virtually mirror-opposite biopsychosocial profiles. Beyond these results, we observed variability among subgroups that was less evident in the aggregate; women who had statistically comparable levels of violence exposure manifested substantially different biopsychosocial profiles.

The results of this and future investigations can inform the development of adaptive interventions for survivors of partner violence that have the potential to be more effective than universal interventions Consistent with many practice standards, adaptive interventions are tailored to an individual's problems and strengths and individuals receive varying amounts of an intervention or varying intervention elements based on their needs and resources, even though the tailoring comes through standardized procedures based on the best available evidence (Collins et al., 2004).

Our findings argue for the benefit of assessing multiple indicators of biopsychosocial functioning. Women with similarly elevated psychological vulnerability related to their battering (e.g., sense of entrapment, danger, powerlessness) may benefit from different kinds of safety planning and therapeutic support based on factors such as level of depression and the relative balance of positive and negative social relationships (as exhibited by Vulnerable-Supported and Vulnerable-Depressed women). Similarly, these findings raise universal support questions such as whether increasing social support necessarily benefits all 
survivors. In some cases, the gap may be so great that some improvement is not enough to improve women's health, safety, and well-being. Furthermore, increasing positive supports may not be sufficient given the undermining effects of these women's negative social relationships. Other indicators, such as impairment in physical functioning, suggest serious problems for which physical or mental health services need to be accessed.

\section{Limitations}

This study provided information about the biopsychosocial heterogeneity among IPV survivors. Although study results can inform tailored and adaptive domestic violence services for IPV survivors, they should be viewed in light of the study's limitations.

Overall, the women who chose not to participate in this study were similar to the women who did participate, with two notable exceptions: nonparticipants were less likely to have taken out protection orders and were more likely to have remained living with the intimate partner who was violent. Such differences highlight the importance, yet difficulty of accessing the full range of IPV victims. We cannot ascertain, for example, whether nonparticipants would be adequately captured within the five groups established here or may represent an additional group. Although the sample was relatively diverse, the small number of specific groups of women of color and ethnicities may also limit the generalizability of our findings.

Readers should also keep in mind that the women who came into this study were recruited based on some kind of legal action. It was not necessary for the woman to make the call to the police herself. Nevertheless, the findings may not generalize to battered women who belong to certain groups (e.g., immigrant communities) or live in particular geographical areas (e.g., rural) where the police may be less likely to become involved with incidents of IPV.

Although every effort was made to select the most parsimonious and best fitting model, two reasons underscore why it is premature to suggest that the groups found in this investigation constitute a complete typology. First, there is a likelihood other models also fit the data. Second, though there is growing attention to and use of the type of person-centered statistical analysis used here, uncertainty exists regarding how best to select models that fit the data (Bauer \& Curran, 2004). Given these limitations, we encourage other violence researchers to use person-centered analyses and methods to investigate the generalizability of these findings across diverse samples. In addition, we encourage future research efforts that will extend this type of analysis to include other relevant biopsychosocial factors.

\section{Conclusion}

Findings that each of the psychosocial factors studied here influence an IPV-exposed woman's capacity to cope, self-protect, and recover are not new. What is new is the portrayal of underlying patterning; that is, how these factors combine across IPV survivors in ways that differentially affect their abilities to resist and cope. Person-oriented research approaches open up new horizons for tackling heterogeneity and complexity within battered women and others affected by violence.

Goodman and Epstein (2008) recently called for a renewed focus in the antidomestic violence movement for survivor-centered, holistic, and comprehensive services. The findings from this study help to provide evidence about how such survivor-centered services should be developed and tailored to address the unique needs of groups of violence survivors. In addition, this study illustrates how violence researchers can use personoriented tools to identify subgroups based on factors that are meaningful to their research question. 
We encourage a commitment to integrative analyses that include person-oriented methodologies that complement and extend variable-oriented approaches, thus refining questions and deepening understanding regarding heterogeneity among battered women and other violence affected populations.

\section{Acknowledgments}

This project was supported by Grants 1 R01 DA11151, "Protection of Women: Health and Justice Outcomes," from the Centers for Disease Control and Prevention, National Institutes of Health, and National Institute of Justice, as part of the Interagency Consortium on Violence Against Women and Family Violence Research and Grant 5 T32 MH200010 "Mental Health Prevention Research Training Program." We acknowledge and appreciate the contributions of Victoria Holt, Mary Kernic, Frederick Rivara, Marsha Wolf, Susan White and Diane Wyant

\section{Funding}

The authors disclosed that they received the following support for their research and/or authorship of this article: The Centers for Disease Control and Prevention, National Institutes of Health, and National Institute of Justice.

\section{References}

Bauer DJ, Curran PJ. The integration of continuous and discrete latent variable models: Potential problems and promising opportunities. Psychological Methods. 2004; 9(1):3-29. [PubMed: 15053717]

Bergman, LR. The application of a person-oriented approach: Types and clusters. In: Bergman, LR.; Cairns, RB.; Nilsson, L.; Nystedt, L., editors. Developmental science and the holistic approach. Mahwah, NJ: Erlbaum; 2000. p. 137-154.

Bergman LR, Trost K. The person-oriented versus the variable-oriented approach: Are they complementary, opposites, or exploring different worlds? Merrill-Palmer Quarterly. 2006; 52:601632.

Bergman, LR.; von Eye, A.; Magnusson, D. Person-oriented research strategies in developmental psychopathology. In: Cicchetti, D.; Cohen, DJ., editors. Developmental psychopathology, 2nd ed. Volume One: Theory and method. New York: Wiley; 2006. p. 850-888.

Bogat GA, Levendosky AA, von Eye A. The future of research on intimate partner violence: Personoriented and variable-oriented perspectives. American Journal of Community Psychology. 2005; 36(1/2):49-70. [PubMed: 16134044]

Bollen KA. Latent variables in psychology and the social science. Annual Review of Psychology. 2002; 53:605-634.

Briere J, Jordan CE. Violence against women: Outcome complexity and implications for assessment and treatment. Journal of Interpersonal Violence. 2004; 19:1252-1276. [PubMed: 15534329]

Bybee DI, Sullivan CM. The process through which an advocacy intervention resulted in positive change for battered women over time. American Journal of Community Psychology. 2002; 30:103132. [PubMed: 11928772]

Campbell JC. Health consequences of intimate partner violence. Lancet. 2002; 359:1331-1336. [PubMed: 11965295]

Carbone-López K, Kruttschnitt C, Macmillan R. Patterns of intimate partner violence and their associations with physical health, psychological distress, and substance abuse. Public Health Reports. 2006 Jul-Aug;121:382-392. [PubMed: 16827439]

Carlson BE, McNutt LA, Choi DY, Rose IM. Intimate partner abuse and mental health. Violence Against Women. 2002; 8:720-745.

Cleland CM, Rothschild L, Haslam N. Detecting latent taxa: Monte Carlo comparison of taxometric, mixture model, and clustering procedures. Psychological Reports. 2000; 87(1):37-47. [PubMed: 11026388]

Coker AL, Smith PH, Bethea L, King M, McKeown R. Physical health consequences of physical and psychological intimate partner violence. Archives of Family Medicine. 2000; 9:451-457. [PubMed: 10810951] 
Coker AL, Smith PH, Fadden MK. Intimate partner violence and disabilities among women attending family practice clinics. Journal of Women's Health. 2005; 14:829-838.

Coker AL, Watkins KW, Smith PH, Brandt HM. Social support reduces the impact of partner violence on health: Application of structural equation models. Preventive Medicine. 2003; 37:259-267. [PubMed: 12914832]

Collins LM, Murphy SA, Bierman KL. A conceptual framework for adaptive preventive interventions. Prevention Science. 2004; 5:185-196. [PubMed: 15470938]

Cooper P, Osborn M, Gath D, Feggetter G. Evaluation of modified self-report measure of social adjustment. British Journal of Psychiatry. 1982; 141:68-75. [PubMed: 7116075]

Curran PJ, Willoughby MT. Implications of latent trajectory models for the study of developmental psychopathology. Development and Psychopathology. 2003; 15:581-612. [PubMed: 14582933]

Davies, J.; Lyon, E.; Monti-Catania, D. Safety planning with battered women: Complex lives/difficult choices. Thousand Oaks, CA: Sage; 1998.

Everitt, BS.; Landau, S.; Morven, L. Cluster analysis. 4th ed.. New York: Oxford University Press; 2001.

Gibson WA. Three multivariate models: Factor analysis, latent structure analysis, and latent profile analysis. Psychometrika. 1959; 24:229-252.

Golding JM. Intimate partner violence as a risk factor for mental disorders: A meta-analysis. Journal of Family Violence. 1999; 14:99-132.

Goodkind J, Sullivan CM, Bybee D. A contextual analysis of battered women's safety planning. Violence Against Women. 2004; 10:514-533.

Goodman, LA.; Epstein, D. Listening to battered women: A survivor-centered approach to advocacy, mental health and justice. Washington DC: American Psychological Association; 2008.

Hagenaars JA, Halman LC. Searching for idea types: The potentialities of latent class analysis. European Sociological Review. 1989; 5(1):81-96.

Hathaway J, Mucci L, Silverman J, Brooks D, Mathews R, Pavlos C. Health status and health care use of Massachusetts women reporting partner abuse. American Journal of Preventive Medicine. 2000; 19:302-307. [PubMed: 11064235]

Hazen A, Connelly C, Kelleher K, Landsverk J, Barth R. Intimate partner violence among female caregivers of children reported for child maltreatment. Child Abuse \& Neglect. 2004; 28:301-319. [PubMed: 15066348]

Henning KR, Klesges LM. Utilization of counseling and supportive services by female victims of domestic abuse. Violence and Victims. 2002; 17:623-636. [PubMed: 12477102]

Hughes HM, Huth-Bocks AC. Variations in parenting stress in African-American battered women: Implications for children's adjustment and family intervention. European Psychologist. 2007; 12(1):62-71.

Hutchison IW, Hirschel JD. Abused women: Help-seeking strategies and police utilization. Violence Against Women. 1998; 4:436-456.

Kemp A, Rawlings EI, Green BL. Post-traumatic stress disorder (PTSD) in battered women: A shelter sample. Journal of Traumatic Stress. 1991; 4:137-148.

Kohl PL, Macy RJ. Profiles of victimized women among the child welfare population: Implications for targeted child welfare policy and practices. Journal of Family Violence. 2008; 23:57-68.

Kramer A, Lorenzon D, Mueller G. Prevalence of intimate partner violence and health implications for women using emergency departments and primary care clinics. Women's Health Issues. 2004; 14:19-29. [PubMed: 15001185]

Lazarus, RS.; Folkman, S. Stress, appraisal, and coping. New York: Springer; 1984.

Lindhorst T, Nurius PS, Macy RJ. Contextualized assessment with battered women: Strategic safety planning to cope with multiple harms. Journal of Social Work Education. 2005; 41:331-352. [PubMed: 18167523]

Logan TK, Walker R, Cole J, Leukefeld C. Victimization and substance abuse among women: Contributing factors, interventions, and implications. Review of General Psychology. 2002; 6:325-397. 
Macy RJ. A research agenda for sexual revictimization: Priority areas and innovative statistical methods. Violence Against Women. 2008; 14:1128-1147. [PubMed: 18685039]

Macy RJ, Nurius PS, Kernic M, Holt V. Battered women's profiles associated with service helpseeking efforts: Illuminating opportunities for intervention. Social Work Research. 2005; 29:137150.

McFarlane J, Parker B, Soeken K, Silva C, Reel S. Safety behaviors of abused women after an intervention during pregnancy. Journal of Obstetric, Gynecologic, and Neonatal Nursing. 1998; 27:64-69.

McNamara JR, Ertl MA, Marsh S, Walker S. Short-term response to counseling and case management intervention in a domestic violence shelter. Psychological Reports. 1997; 81:1243-1251. [PubMed: 9461760]

McNutt L, Carlson B, Persaud M, Postmus J. Cumulative abuse experiences, physical health and health behaviours. Annals of Epidemiology. 2002; 12:123-130. [PubMed: 11880220]

Muthen BO. Beyond SEM: General latent variable modeling. Behaviormetrika. 2002; 29(1):81-117.

Nurius, PS.; Macy, RM. Escape and protection differences among battered women; Paper Presented at the Society for Social Work \& Research Conference; San Antonio, TX. 2006 Jan 13.

Nurius PS, Macy RJ. Heterogeneity among violence exposed women: Applying person-oriented research methods. Journal of Interpersonal Violence. 2008; 23:389-415. [PubMed: 18245574]

Nurius PS, Macy RJ, Bhuyan R, Holt V, Kernic M, Rivara F. Depression and physical functioning of battered women: Adding vulnerability and resources to the analysis. Journal of Interpersonal Violence. 2003; 18:1411-1431. [PubMed: 14678614]

Pape KT, Arias I. The role of perceptions and attiributions in battered women's intentions to permanently end their violent relationships. Cognitive Therapy and Research. 2000; 24:201-214.

Plichta SB. Intimate partner violence and physical health consequences: Policy and practice implications. Journal of Interpersonal Violence. 2004; 19:1296-1323. [PubMed: 15534333]

Plitcha SB, Falik M. Prevalence of violence and its implications for women's health. Women's Health Issues. 2001; 11:244-258. [PubMed: 11336864]

Porcerelli JH, Cogan R, West PP, Rose EA, Lambrecht D, Wilson KE, et al. Violent victimization of women and men: Physical and psychiatric symptoms. Journal of American Board of Family Practice. 2003; 16:32-39.

Riger S, Raja S, Camacho J. The radiating impact of intimate partner violence. Journal of Interpersonal Violence. 2002; 17:184-205.

Seng JS. A conceptual framework for research on lifetime violence, posttraumatic stress, and childbearing. Journal of Midwifery and Women's Health. 2002; 47:337-346.

Smith PH, Earp JAL, DeVellis R. Measuring battering: Development of the women's experience with battering (WEB) scale. Women's Health: Research on gender, Behavior, and Policy. 1995; 1:273288.

Smith PH, Smith JB, Earp JAL. Beyond the measurement trap a reconceptualization and measurement of woman battering. Psychology of Women Quarterly. 1999; 23:177-193.

Smith PH, Thornton GE, DeVellis R, Earp J, Coker AL. A population-based study of the prevalence and distinctiveness of battering, physical assault, and sexual assault in intimate relationships. Violence Against Women. 2002; 8:1208-1232.

Smith, SP.; Edwards, G.; DeVellis, R. Intimate partner violence: Prevalence, co-occurrence, and health consequences. Washington, DC: American Public Health Association; 1998.

Stevens, J. Applied multivariate statistics for the social science. 3rd ed.. Mahwah, NJ: Lawrence Erlbaum; 1996.

Straus MA, Hamby SL, Boney McCoy S, Sugarman DB. The revised Conflict Tactics Scales (CTS2): Development and preliminary psychometric data. Journal of Family Issues. 1996; 17:283-316.

Sutherland CA, Bybee D, Sullivan CM. Beyond bruises and broken bones: The joint effects of stress and injuries on battered women's health. American Journal of Community Psychology. 2002; 30:609-636. [PubMed: 12188053] 
Taylor SE, Klein LC, Lewis BP, Gruenewald TL, Gurung RAR, Updegraff JA. Biobehavioral responses to stress in females: Tend-and-befriend, not fight-or-flight. Psychological Review. 2000; 107:411-429. [PubMed: 10941275]

Tjaden P, Thoennes N. Prevalence and consequences of male-to-female and female-to-male intimate partner violence as measured by the National Violence Against Women Survey. Violence Against Women. 2000; 6:142-161.

Tjaden, P.; Thoennes, N. Extent, nature and consequences of rape victimization: Findings from the National Violence Against Women Survey. Washington, DC: National Institute of Justice; 2006. (NCJ 210346).

Tolman RM, Wang H. Domestic violence and women's employment: Fixed effects models of three waves of women's employment study data. American Journal of Community Psychology. 2005; 36:147-158. [PubMed: 16134051]

von Eye A, Bogat GA. Person-oriented and variable-oriented research: Concepts, results, and development. Merrill-Palmer Quarterly. 2006; 52:390-420.

von Eye A, Bogat GA, Rhodes JE. Variable-oriented and person-oriented perspectives of analysis: The example of alcohol consumption in adolescence. Journal of Adolescence. 2007; 29:981-1004. [PubMed: 17045640]

Ware JE, Kosinski M, Bayliss MS, McHorney CA, Rogers WH, Raczek A. Comparison of methods for the scoring and statistical analysis of SF-36 health profile and summary measures: Summary of results from the medical outcomes study. Medical Care. 1995; 33(Suppl. 4):264-279.

Wasco SM, Campbell R, Howard A, Mason GE, Staggs SL, Schewe PA, et al. A statewide evaluation of services provided to rape survivors. Journal of Interpersonal Violence. 2004; 19:252-263. [PubMed: 15011687]

Weissman MM, Prusoff BA, Thompson WD, Harding PS, Myers JK. Social adjustment by self-report in a community sample and in psychiatric outpatients. Journal of Nervous and Mental Disease. 1978; 166:317-326. [PubMed: 650195]

Wolf ME, Holt VL, Kernic MA, Rivara FP. Who gets protection orders for intimate partner violence? American Journal of Preventive Medicine. 2000; 19:286-291. [PubMed: 11064233]

Zlotnick C, Johnson D, Kohn R. Intimate partner violence and longterm psychosocial functioning in a national sample of American women. Journal of Interpersonal Violence. 2006; 21:262-275. [PubMed: 16368765]

\section{Biographies}

Paula S. Nurius, PhD, MSW, MA, is a professor and the director of the NIMH-funded Prevention Research Training Program at the University of Washington School of Social Work where she has been on faculty since 1984. Her graduate degrees are in psychology and social welfare from the universities of Hawaii and Michigan. Her practice background is in women's services and trauma exposure (mental health, violence, sexuality). She has integrated perspectives from social psychology, social work, and public health in social cognitive analysis of self-concept development and functioning and on cognitive-affective processes under conditions of stress and vulnerability. Her current research focuses on women's coping responses to relationship violence, risk and protective factors impinging on coping and protective action, and preventive interventions designed to strengthen resistance and resilience. She is also examining the effects of differential traumatic stress exposure histories among high risk youth, charting trajectories of multilevel problem development or resilience in the transition from adolescence to early adulthood.

Rebecca J. Macy, PhD, ACSW, LCSW, is an associate professor at the School of Social Work at the University of North Carolina at Chapel Hill and a core faculty member of the UNC Injury Prevention Research Center. She joined the faculty in 2002 after receiving her doctoral degree in social welfare from the University of Washington in Seattle. In 1993, she received her MSW from Tulane University in New Orleans. She is a licensed social worker with practice experience in community mental health. Her current research activities focus 
on the physical and mental health consequences of violent victimization, repeated victimizations across the life span, processes for coping with traumatic events, the development of violence prevention and interventions for violence survivors, communitybased services for domestic violence and sexual assault survivors, and the application of advanced statistical methods to the study of violence and trauma. She is also interested in the application of social psychological and cognitive theories toward understanding the mental health consequences of violent victimization and has written about cognitive therapy techniques. She teaches course on social work practice, family violence, and statistics. 


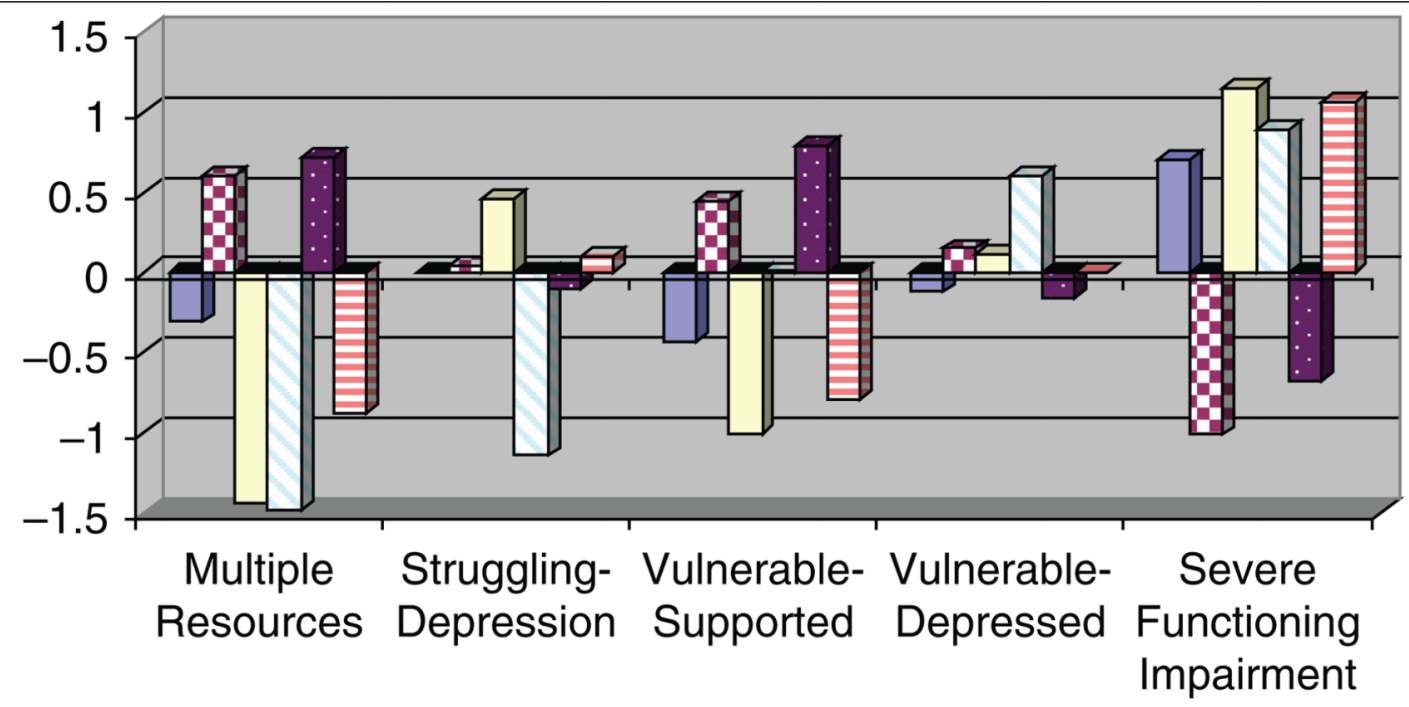

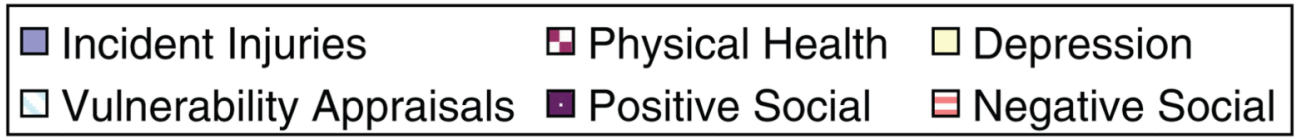

Figure 1.

Standardized profile group means for biopsychosocial variables 


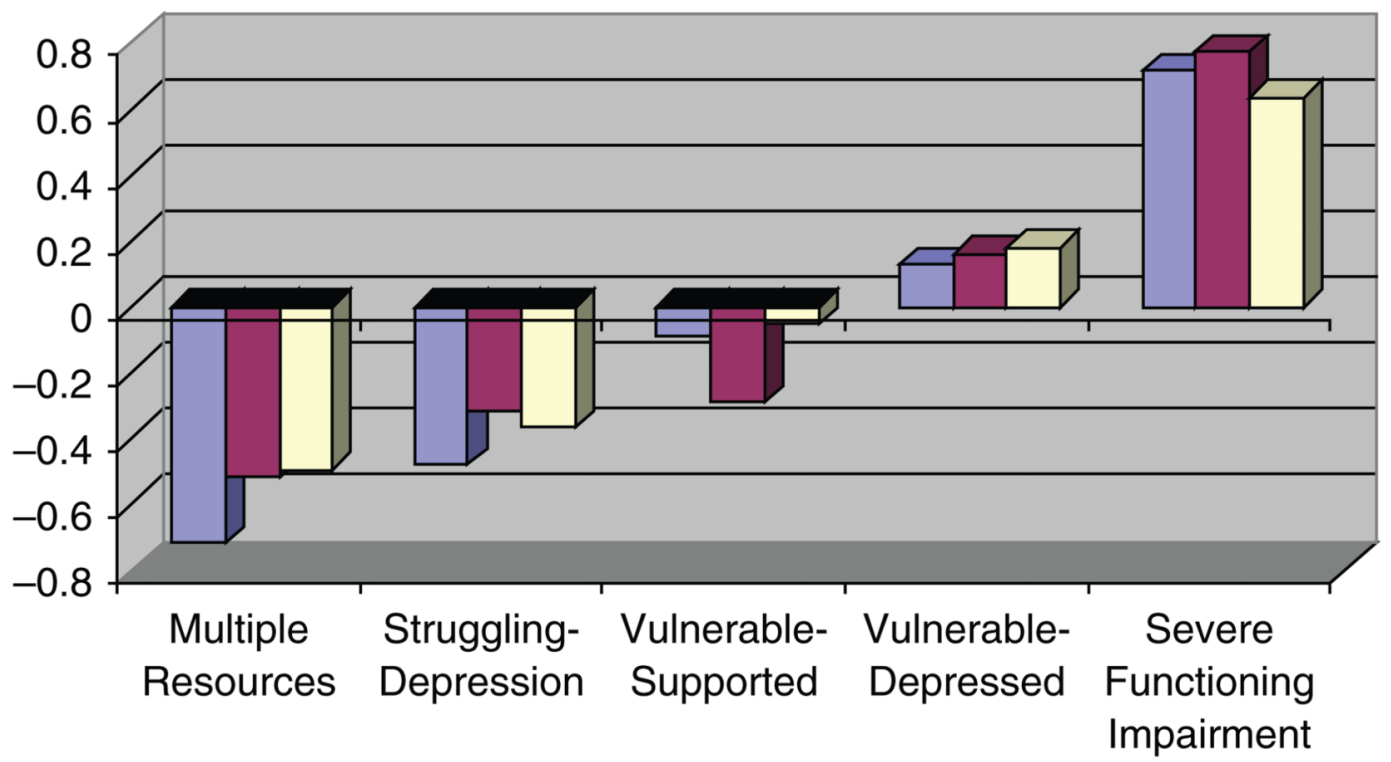

\section{$\square$ Psychological Abuse $\square$ Physical Abuse $\square$ Sexual Abuse}

Figure 2.

Standardized intimate partner violence means for past year among 


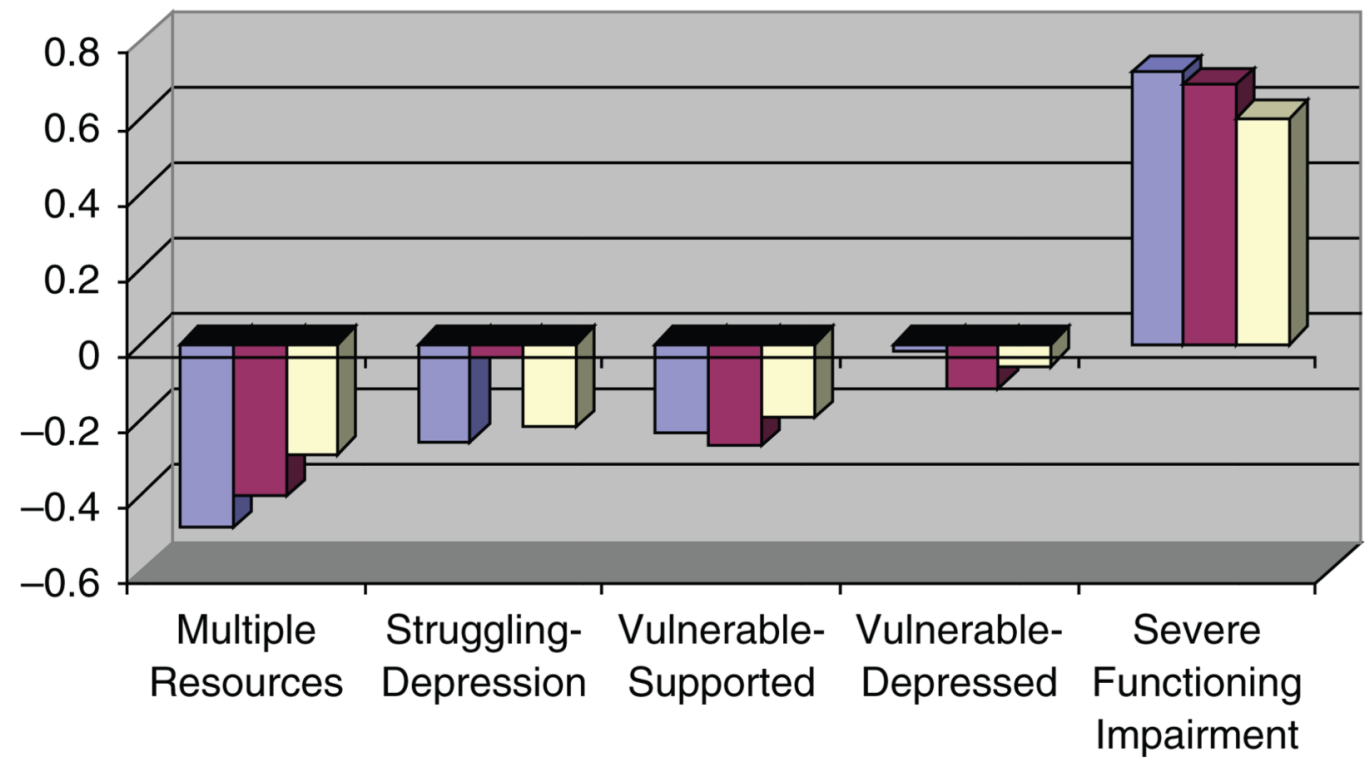

\section{$\square$ Psychological Abuse $\square$ Physical Abuse $\square$ Sexual Abuse}

Figure 3.

Standardized intimate partner violence means at incident among profile groups 


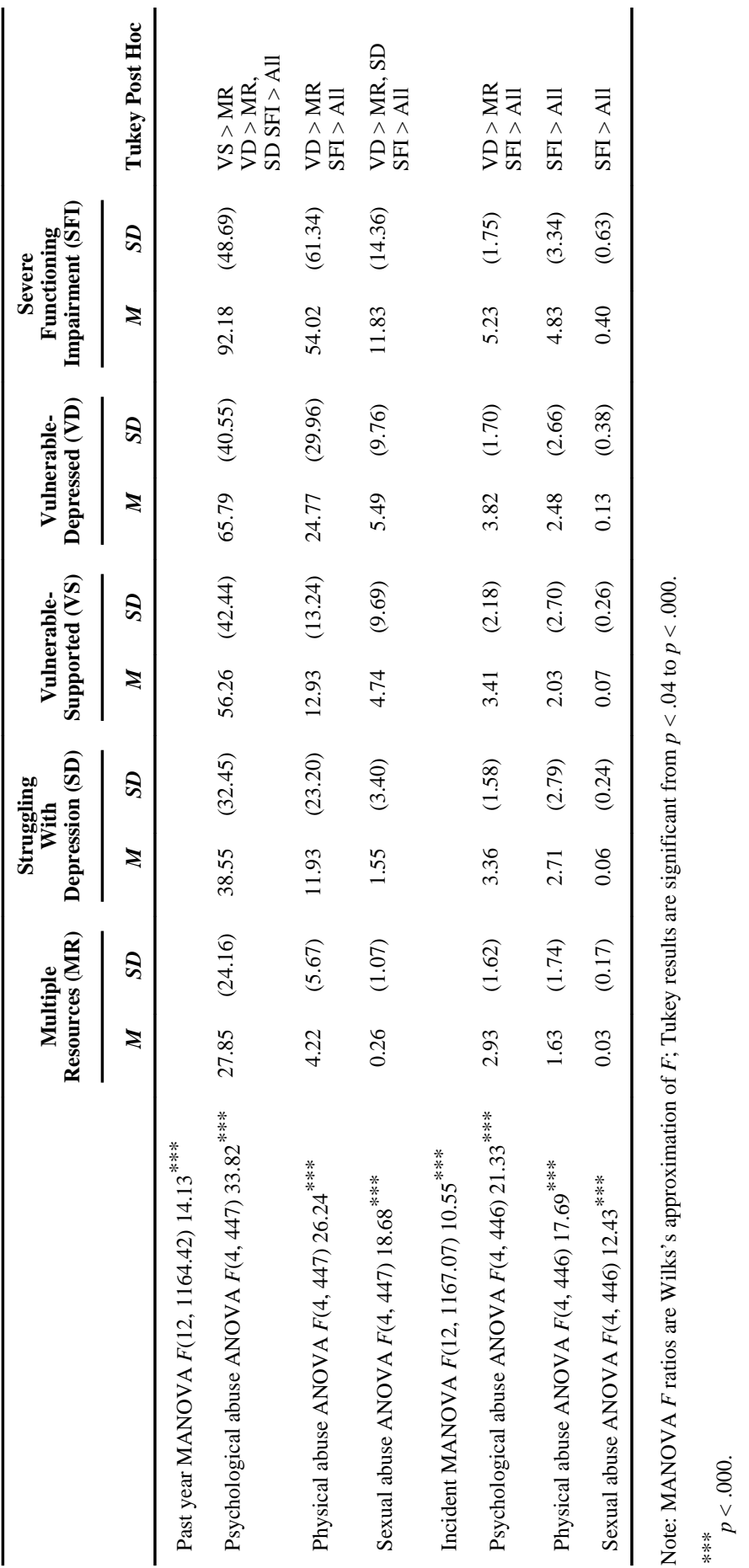

